\title{
Coordinate Independence of of Quantum-Mechanical Path Integrals
}

\author{
H. Kleinert* and A. Chervyakov ${ }^{\dagger}$ \\ Freie Universität Berlin \\ Institut für Theoretische Physik \\ Arnimallee14, D-14195 Berlin
}

\begin{abstract}
We develop simple rules for performing integrals over products of distributions in coordinate space. Such products occur in perturbation expansions of path integrals in curvilinear coordinates, where the interactions contain terms of the form $\dot{q}^{2} q^{n}$, which give rise to highly singular Feynman integrals. The new rules ensure the invariance of perturbatively defined path integrals under coordinate transformations.
\end{abstract}

\section{INTRODUCTION}

In the previous papers [1,2], we have presented a diagrammatic proof of reparametrization invariance of perturbatively defined quantum-mechanical path integrals. The proper perturbative definition of path integrals was shown to require an extension to a functional integral in $D$ spacetime, and a subsequent analytic continuation to $D=1$. In Ref. [1] the perturbative calculations were performed in momentum space, where Feynman integrals in a continuous number of dimensions $D$ are known from the prescriptions of 't Hooft and M. Veltman [3]. In Ref. [2] we have found the same results directly from the Feynman integrals in the $1-\varepsilon$-dimensional time space with the help of the Bessel representation of Green functions. The coordinate space calculation is interesting for many applications, for instance, if one wants to obtain the effective action of a field system in curvilinear coordinates, where the kinetic term depends on the dynamic variable. Then one needs rules for performing temporal integrals over Wick contractions of local fields.

In this note we want to show that the reparametrization invariance of perturbatively defined quantummechanical path integrals can be obtained in the coordinate space with the help of a simple but quite general arguments based on the inhomogeneous field equation for the Green function, and rules of the partial integration. The prove does not require the calculation of the Feynman integrals separately and remains valid for the functional integrals in an arbitrary space-time dimension D.

\footnotetext{
*E-mail: kleinert@physik.fu-berlin.de

${ }^{\dagger}$ On leave from LCTA, JINR, Dubna, Russia E-mail: chervyak@physik.fu-berlin.de
}

\section{PROBLEM WITH COORDINATE TRANSFORMATIONS}

Recall the origin of the difficulties with coordinate transformations in path integrals. Let $x(\tau)$ be the euclidean coordinates of a quantum-mechanical point particle of unit mass in a harmonic potential $\omega^{2} x^{2} / 2$ as a function of the imaginary time $\tau=-i t$. Under a coordinate transformation $x(\tau) \rightarrow q(\tau)$ defined by $x(\tau)=f(q(\tau))=q(\tau)+\sum_{n=2}^{\infty} a_{n} q^{n}(\tau)$, the kinetic term $\dot{x}^{2}(\tau) / 2$ goes over into $\dot{q}^{2}(\tau) f^{\prime 2}(q(\tau)) / 2$. If the path integral over $q(\tau)$ is performed perturbatively, the expansion terms contains temporal integrals over Wick contractions which, after suitable partial integrations, are products of the following basic correlation functions

$$
\begin{aligned}
\Delta\left(\tau-\tau^{\prime}\right) & \equiv\left\langle q(\tau) q\left(\tau^{\prime}\right)\right\rangle=- \\
\partial_{\tau} \Delta\left(\tau-\tau^{\prime}\right) & \equiv\left\langle\dot{q}(\tau) q\left(\tau^{\prime}\right)\right\rangle=\ldots .- \\
\partial_{\tau} \partial_{\tau^{\prime}} \Delta\left(\tau-\tau^{\prime}\right) & \equiv\left\langle\dot{q}(\tau) \dot{q}\left(\tau^{\prime}\right)\right\rangle=\ldots \ldots . .
\end{aligned}
$$

The right-hand sides define the line symbols to be used in Feynman diagrams for the interaction terms.

Explicitly, the first correlation function reads

$$
\Delta\left(\tau, \tau^{\prime}\right)=\frac{1}{2 \omega} e^{-\omega\left|\tau-\tau^{\prime}\right|} .
$$

The second correlation function (2) has a discontinuity

$$
\partial_{\tau} \Delta\left(\tau, \tau^{\prime}\right)=-\frac{1}{2} \epsilon\left(\tau-\tau^{\prime}\right) e^{-\omega\left|\tau-\tau^{\prime}\right|},
$$

where

$$
\epsilon\left(\tau-\tau^{\prime}\right) \equiv 2 \int_{-\infty}^{\tau} d \tau^{\prime \prime} \delta\left(\tau^{\prime \prime}-\tau^{\prime}\right)
$$

is a distribution which has a jump at $\tau=\tau^{\prime}$. The third correlation function (3) contains a $\delta$-function:

$$
\partial_{\tau} \partial_{\tau^{\prime}} \Delta\left(\tau, \tau^{\prime}\right)=\delta\left(\tau-\tau^{\prime}\right)-\frac{\omega}{2} e^{-\omega\left|\tau-\tau^{\prime}\right|},
$$

The temporal integrals in $\tau$-space over products of such distributions are undefined.

In our previous papers [1]2] we have shown that a unique perturbation expansions leading to a reparametrization invariant theory is obtained by extending the path integral to a $D$-dimensional functional integral, and by performing the perturbation expansion in $D$-dimensional space, with a limit $D \rightarrow 1$ taken at the end.

In this note we shall set up simple rules for integrals over products of the correlation functions in the extended coordinate space the same results. 


\section{MODEL SYSTEM}

To be specific, we shall prove the coordinate independence of the exactly solvable path integral of a point particle of unit mass in a harmonic potential $\omega^{2} x^{2} / 2$, over a large imaginary-time interval $\beta$,

$$
Z_{\omega}=\int \mathcal{D} x(\tau) e^{-\mathcal{A}_{\omega}[x]}=e^{-\operatorname{Tr} \log \left(-\partial^{2}+\omega^{2}\right)}=e^{-\beta \omega / 2} .
$$

The action is

$$
\mathcal{A}_{\omega}=\frac{1}{2} \int d \tau\left[\dot{x}^{2}(\tau)+\omega^{2} x^{2}(\tau)\right] .
$$

A coordinate transformation turns (\$) into a path integral with a singular perturbation expansion.

For simplicity we assume the coordinate transformation to preserve the symmetry $x \leftrightarrow-x$ of the initial oscillator, such its power series expansion starts out like $x(\tau)=f(q(\tau))=q-g q^{3} / 3+g^{2} a q^{5} / 5-\cdots$, where $g$ is a smallness parameter, and $a$ an extra parameter. We shall see that the perturbation expansion is independent of $a$, such that $a$ will merely serve to check the calculations. The transformation changes the partition function (8) into

$$
Z=\int \mathcal{D} q(\tau) e^{-\mathcal{A}_{J}[q]} e^{-\mathcal{A}[q]},
$$

where the transformed action $\mathcal{A}[q]=\mathcal{A}_{\omega}[q]+\mathcal{A}_{\text {int }}[q]$ is decomposed into a free part

$$
\mathcal{A}_{\omega}[q]=\frac{1}{2} \int d \tau\left[\dot{q}^{2}(\tau)+\omega^{2} q^{2}(\tau)\right]
$$

and an interacting part, which reads to second order in $g$ :

$$
\begin{aligned}
& \mathcal{A}_{\text {int }}[q]=\frac{1}{2} \int d \tau\left\{-g\left[2 \dot{q}^{2}(\tau) q^{2}(\tau)+\frac{2 \omega^{2}}{3} q^{4}(\tau)\right]\right. \\
& \left.+g^{2}\left[(1+2 a) \dot{q}^{2}(\tau) q^{4}(\tau)+\omega^{2}\left(\frac{1}{9}+\frac{2 a}{5}\right) q^{6}(\tau)\right]\right\} .
\end{aligned}
$$

The exponent in (10) contains an additional effective action $\mathcal{A}_{J}[q]$ coming from the Jacobian of the coordinate transformation:

$$
\mathcal{A}_{J}[q]=-\delta(0) \int d \tau \log \frac{\delta f(q(\tau))}{\delta q(\tau)} .
$$

This has the power series expansion

$$
\mathcal{A}_{J}[q]=-\delta(0) \int d \tau\left[-g q^{2}(\tau)+g^{2}\left(a-\frac{1}{2}\right) q^{4}(\tau)\right] .
$$

For $g=0$, the transformed partition function (10) coincides with (8). When expanding $Z$ of Eq. (10) in powers of $g$, we obtain a sum of Wick contractions with associated Feynman diagrams contributing to each order $g^{n}$.
This sum must vanish to ensure coordinate invariance of the path integral.

By considering only connected Feynman diagrams, we shall obtain an expansion for the free energy

$$
F=F_{\omega}+\sum_{n=1} g^{n} F_{n}
$$

where $F_{\omega}$ is the free energy of the unperturbed harmonic oscillator (8). The coordinate invariance is ensured by the vanishing of all expansion terms $F_{n}$.

\section{EXPANSION TERMS OF FREE ENERGY DENSITY}

The graphical expansion for the ground state energy will be carried here only up to three loops. The diagrams are composed of the three types of lines in (11)-(3), and new interaction vertices for each power of $g$. The diagrams coming from the Jacobian action (14) are easily recognized by an accompanying power of $\delta(0)$.

To lowest order in $g$, there exists only three diagrams, two originated from the interaction (12), one from the Jacobian action (14):

$$
-F_{1}=-g\left[\bigcirc+\omega^{2} \bigcirc-\delta(0) \bigcirc\right] \text {. }
$$

To order $g^{2}$, we distinguish several contributions. First there are two three-loop local diagrams coming from the interaction (12), and one two-loop local diagram from the Jacobian action (14):

$$
\begin{array}{rl}
-F_{2}^{(1)}=g^{2} & 3\left(\frac{1}{2}+a\right) \circlearrowleft+15 \omega^{2}\left(\frac{1}{18}+\frac{a}{5}\right) \searrow \\
& -3\left(a-\frac{1}{2}\right) \delta(0) \bigcirc
\end{array}
$$

We call a diagram local if it involves no temporal time integral. The Jacobian action (14) contributes further the nonlocal diagrams:

$$
\begin{aligned}
-F_{2}^{(2)}= & -\frac{g^{2}}{2 !}\left\{2 \delta^{2}(0) \bigcirc\right. \\
& \left.-4 \delta(0)\left[\bigcirc+\bigcirc+2 \omega^{2} \bigcirc\right]\right\} .
\end{aligned}
$$

In the perturbative calculations to follow, we shall use dimensional regularization where $\delta(0)=0$, according to a basic rule of t'Hooft and Veltman [3]. As a consequence, the last terms in $F_{1}, F_{2}^{(1)}$, and the entire $F_{2}^{(2)}$ are zero. In fact, the term $\mathcal{A}_{J}[q]$ may be omitted completely from the path integral (10).

The remaining diagrams are either of the three-bubble type, or of the watermelon type, each with all possible 
combinations of the three line types (1)-(3). The former are

$$
\begin{aligned}
& -F_{2}^{(3)}=-\frac{g^{2}}{2 !}\left[\begin{array}{ll}
4 & \bigcirc
\end{array}\right.
\end{aligned}
$$

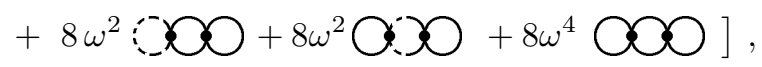

and the latter:

$$
-F_{2}^{(4)}=-\frac{g^{2}}{2 !} 4\left[\Theta+4 \Leftrightarrow+\Leftrightarrow+4 \omega^{2} \oiint+\frac{2}{3} \omega^{4} \supseteqq\right] \text {. }
$$

Since the equal-time expectation value $\langle\dot{q}(\tau) q(\tau)\rangle$ vanishes by Eq. (5), diagrams with a local contraction of a mixed line (2) are trivially zero, and have been omitted.

In our previous papers [1]:2], all integrals were calculated individually in $D=1-\varepsilon$ dimensions, taking the limit $\varepsilon \rightarrow 0$ at the end. Here we set up simple rules for finding the same results, which make the sum of all Feynman diagrams contributing to each order $g^{n}$ vanish.

\section{BASIC PROPERTIES OF DIMENSIONALLY REGULARIZED DISTRIBUTIONS}

The path integral (10) is extended to an associated functional integral in a $D$-dimensional coordinate space $x$, with coordinates $x_{\mu} \equiv\left(\tau, x_{2}, x_{3}, \ldots\right)$, by replacing $\dot{q}^{2}(\tau)$ in the kinetic term by $\left(\partial_{\mu} q(x)\right)^{2}$, where $\partial_{\mu}=$ $\partial / \partial x_{\mu}$. The Jacobian action term (13) is omitted in dimensional regularization because of Veltman's rule [3]:

$$
\delta^{(D)}(0)=\int \frac{d^{D} k}{(2 \pi)^{D}}=0 .
$$

In our calculations, we shall encounter generalized $\delta$ functions, which are multiple derivatives of the ordinary $\delta$-function:

$$
\begin{aligned}
\delta_{\mu_{1} \ldots \mu_{n}}^{(D)}(x) & \equiv \partial_{\mu_{1} \ldots \mu_{n}} \delta^{(D)}(x) \\
& =\int d^{D} k(i k)_{\mu_{1}} \ldots(i k)_{\mu_{n}} e^{i k x},
\end{aligned}
$$

with $\partial_{\mu_{1} \ldots \mu_{n}} \equiv \partial_{\mu_{1}} \ldots \partial_{\mu_{n}}$, and with $d^{D} k \equiv d^{D} k /(2 \pi)^{D}$. In dimensional regularization, alle these vanish at the origin as well:

$$
\delta_{\mu_{1} \ldots \mu_{n}}^{(D)}(0)=\int d^{D} k(i k)_{\mu_{1}} \ldots(i k)_{\mu_{n}}=0
$$

which is a more general way of expressing Veltman's rule. In the extended coordinate space, the correlation function (11) becomes

$$
\Delta(x)=\int \frac{d^{D} k}{(2 \pi)^{D}} \frac{e^{i k x}}{k^{2}+\omega^{2}}
$$

At the origin, it has the value

$$
\Delta(0)=\int \frac{d^{D} k}{k^{2}+\omega^{2}}=\frac{\omega^{D-2}}{(4 \pi)^{D / 2}} \Gamma\left(1-\frac{D}{2}\right) \underset{D=1}{=} \frac{1}{2 \omega} .
$$

The extension of the time derivative (2),

$$
\Delta_{\mu}(x)=\int \vec{d}^{D} k \frac{i k_{\mu}}{k^{2}+\omega^{2}} e^{i k x}
$$

vanishes at the origin, $\Delta_{\mu}(0)=0$. This follows directly from a Taylor series expansion of $1 /\left(k^{2}+\omega^{2}\right)$ in powers of $k^{2}$, together with Eq. 23).

The second derivative of $\Delta(x)$ has the Fourier representation

$$
\Delta_{\mu \nu}(x)=-\int d^{D} k \frac{k_{\mu} k_{\nu}}{k^{2}+\omega^{2}} e^{i k x}
$$

Contracting the indices yields

$$
\Delta_{\mu \mu}(x)=-\int d^{D} k \frac{k^{2}}{k^{2}+\omega^{2}} e^{i k x}=-\delta^{(D)}(x)+\omega^{2} \Delta(x),
$$

which follows from the definition of the correlation function by the inhomogeneous field equation

$$
\left(-\partial_{\mu}^{2}+\omega^{2}\right) q(x)=\delta^{(D)}(x)
$$

From (28) we have the relation between integrals

$$
\int d^{D} x \Delta_{\mu \mu}(x)=-1+\omega^{2} \int d^{D} x \Delta(x),
$$

Inserting Veltman's rule (21) into (28), we obtain

$$
\Delta_{\mu \mu}(0)=\omega^{2} \Delta(0) \underset{D=1}{=} \frac{\omega}{2} \text {. }
$$

This ensures the vanishing of the first-order contribution (16) to the free energy

$$
-F_{1}=-g\left[-\Delta_{\mu \mu}(0)+\omega^{2} \Delta(0)\right] \Delta(0)=0 .
$$

The same equation (28) allows us to calculate immediately the second-order contribution (17) from the local diagrams

$$
\begin{aligned}
-F_{2}^{(1)}= & -3 g^{2}\left[\left(\frac{1}{2}+a\right) \Delta_{\mu \mu}(0)-5\left(\frac{1}{18}+\frac{a}{5}\right) \omega^{2} \Delta(0)\right] \\
& \times \Delta^{2}(0)=-\frac{2}{3} \omega^{2} \Delta^{3}(0) \underset{D \rightarrow 1}{=}-\frac{1}{12 \omega} .
\end{aligned}
$$

The other contributions to the free energy in the expansion (15) require rules for calculating products of two and four distributions, which we are now going to develop. 


\section{INTEGRALS OVER PRODUCTS OF TWO DISTRIBUTIONS}

The simplest integrals of this type are

$$
\begin{aligned}
& \int d^{D} x \Delta^{2}(x)=\int d^{D} p \vec{d}^{D} k \frac{\delta^{(D)}(k+p)}{\left(p^{2}+\omega^{2}\right)\left(k^{2}+\omega^{2}\right)} \\
& =\int \frac{d^{D} k}{\left(k^{2}+\omega^{2}\right)^{2}}=\frac{\omega^{D-4}}{(4 \pi)^{D / 2}} \Gamma\left(2-\frac{D}{2}\right)=\frac{(2-D)}{2 \omega^{2}} \Delta(0),
\end{aligned}
$$

and

$$
\begin{gathered}
\int d^{D} x \Delta_{\mu}^{2}(x)=-\int d^{D} x \Delta(x)\left[-\delta^{(D)}(x)+\omega^{2} \Delta(x)\right] \\
=\Delta(0)-\omega^{2} \int d^{D} x \Delta^{2}(x)=\frac{D}{2} \Delta(0) .
\end{gathered}
$$

To obtain the second result we have perfomed a partial integration and used (28).

In contrast to the integrals (34) and (35), the integral

$$
\begin{gathered}
\int d^{D} x \Delta_{\mu \nu}^{2}(x)=\int \vec{d}^{D} p \vec{d}^{D} k \frac{(k p)^{2} \delta^{(D)}(k+p)}{\left(k^{2}+\omega^{2}\right)\left(p^{2}+\omega^{2}\right)} \\
=\int \vec{d}^{D} k \frac{\left(k^{2}\right)^{2}}{\left(k^{2}+\omega^{2}\right)^{2}}=\int d^{D} x \Delta_{\mu \mu}^{2}(x)
\end{gathered}
$$

diverges formally in $D=1$ dimension. In dimensional regularization, however, we may decompose $\left(k^{2}\right)^{2}=$ $\left(k^{2}+\omega^{2}\right)^{2}-2 \omega^{2}\left(k^{2}+\omega^{2}\right)+\omega^{4}$, and use (23) to evaluate further

$$
\begin{aligned}
& \int d^{D} x \Delta_{\mu \mu}^{2}(x)=\int \vec{d}^{D} k \frac{\left(k^{2}\right)^{2}}{\left(k^{2}+\omega^{2}\right)^{2}}=-2 \omega^{2} \int \frac{\vec{d}^{D} k}{\left(k^{2}+\omega^{2}\right)} \\
& +\omega^{4} \int \frac{d^{D} k}{\left(k^{2}+\omega^{2}\right)^{2}}=-2 \omega^{2} \Delta(0)+\omega^{4} \int d^{D} x \Delta^{2}(x) .
\end{aligned}
$$

Together with (34), we obtain the finite integrals

$$
\begin{gathered}
\int d^{D} x \Delta_{\mu \nu}^{2}(x)=\int d^{D} x \Delta_{\mu \mu}^{2}(x)=-2 \omega^{2} \Delta(0) \\
+\omega^{4} \int d^{D} x \Delta^{2}(x)=-(1+D / 2) \omega^{2} \Delta(0) .
\end{gathered}
$$

An alternative way of deriving the equality (36) is to use partial integrations and the identity

$$
\partial_{\mu} \Delta_{\mu \nu}(x)=\partial_{\nu} \Delta_{\mu \mu}(x),
$$

which follows directly from the Fourier representation (26).

Finally, from Eqs. (34), (35), and (38), we observe the useful identity

$$
\int d^{D} x\left[\Delta_{\mu \nu}^{2}(x)+2 \omega^{2} \Delta_{\mu}^{2}(x)+\omega^{4} \Delta^{2}(x)\right]=0,
$$

which together with the inhomogeneous field equation (28) reduces the calculation of the second-order contribution of all three-bubble diagrams (19) to zero:

$$
\begin{aligned}
& -F_{2}^{(3)}=-g^{2} \Delta^{2}(0) \\
& \times \int d^{D} x\left[\Delta_{\mu \nu}^{2}(x)+2 \omega^{2} \Delta_{\mu}^{2}(x)+\omega^{4} \Delta^{2}(x)\right]=0 .
\end{aligned}
$$

\section{INTEGRALS PRODUCTS OF FOUR DISTRIBUTIONS}

More delicate integrals arise from the watermelon diagrams in (20) which contain products of four distributions, a nontrivial tensorial structure, and overlapping divergences [1, 2]. Consider the first three diagrams:

$$
\begin{aligned}
\Theta & =\int d^{D} x \Delta^{2}(x) \Delta_{\mu \nu}^{2}(x) . \\
4 & =4 \int d^{D} x \Delta(x) \Delta_{\mu}(x) \Delta_{\nu}(x) \Delta_{\mu \nu}(x), \\
\Leftrightarrow & =\int d^{D} x \Delta_{\mu}(x) \Delta_{\mu}(x) \Delta_{\nu}(x) \Delta_{\nu}(x),
\end{aligned}
$$

To exhibit the subtleties with the tensorial structure, we introduce the integral

$$
I_{D}=\int d^{D} x \Delta^{2}(x)\left[\Delta_{\mu \nu}^{2}(x)-\Delta_{\mu \mu}^{2}(x)\right] .
$$

In $D=1$ dimension, the bracket vanishes formally, but the limit $D \rightarrow 1$ of the integral is nevertheless finite. We now decompose the Feynman diagram (42), into the sum

$$
\int d^{D} x \Delta^{2}(x) \Delta_{\mu \nu}^{2}(x)=\int d^{D} x \Delta^{2}(x) \Delta_{\mu \mu}^{2}(x)+I_{D} .
$$

To obtain an analogous decompositions for the other two diagrams (43) and (44) we derive a few useful relations using the inhomogeneous field equation (28), partial integrations, and Veltman's rule (23). First there is the relation

$$
-\int d^{D} x \Delta_{\mu \mu}(x) \Delta^{3}(x)=\Delta^{3}(0)-\omega^{2} \int d^{D} x \Delta^{4}(x) .
$$

By a partial integration, the left-hand side becomes

$$
\int d^{D} x \Delta_{\mu \mu}(x) \Delta^{3}(x)=-3 \int d^{D} x \Delta_{\mu}^{2}(x) \Delta^{2}(x),
$$

leading to

$$
\int d^{D} x \Delta_{\mu}^{2}(x) \Delta^{2}(x)=\frac{1}{3} \Delta^{3}(0)-\frac{1}{3} \omega^{2} \int d^{D} x \Delta^{4}(x) .
$$

Invoking once more the inhomogeneous field equation (28) and Veltman's rule (21), we obtain the integrals

$$
\int d^{D} x \Delta_{\mu \mu}^{2}(x) \Delta^{2}(x)=-2 \omega^{2} \Delta^{3}(0)+\omega^{4} \int d^{D} x \Delta^{4}(x),
$$

and 


$$
\int d^{D} x \Delta_{\mu \mu}(x) \Delta_{\mu}^{2}(x) \Delta(x)=\omega^{2} \int d^{D} x \Delta_{\mu}^{2}(x) \Delta^{2}(x)
$$

Due to Eq. (49), the integral (51) takes the form

$$
\begin{aligned}
\int d^{D} x \Delta_{\mu \mu}(x) \Delta_{\mu}^{2}(x) \Delta(x) & =\frac{1}{3} \omega^{2} \Delta^{3}(0) \\
& -\frac{1}{3} \omega^{4} \int d^{D} x \Delta^{4}(x) .
\end{aligned}
$$

Partial integration, together with Eqs. (50) and (52), leads to

$$
\begin{aligned}
& \int d^{D} x \partial_{\mu} \Delta_{\lambda \lambda}(x) \Delta_{\mu}(x) \Delta^{2}(x)= \\
& -\int d^{D} x \Delta_{\lambda \lambda}^{2}(x) \Delta^{2}(x)-2 \int d^{D} x \Delta_{\lambda \lambda}(x) \Delta_{\mu}^{2}(x) \Delta(x) \\
& =\frac{4}{3} \omega^{2} \Delta^{3}(0)-\frac{1}{3} \omega^{4} \int d^{D} x \Delta^{4}(x)
\end{aligned}
$$

A further partial integration, and use of Eqs. (39), 51), and (53), produces the decompositions of the second and third Feynman diagrams (43) and (44):

$$
\begin{aligned}
& 4 \int d^{D} x \Delta(x) \Delta_{\mu}(x) \Delta_{\nu}(x) \Delta_{\mu \nu}(x)= \\
& \quad=-2 I_{D}+4 \omega^{2} \int d^{D} x \Delta^{2}(x) \Delta_{\mu}^{2}(x),
\end{aligned}
$$

and

$$
\begin{aligned}
& \int d^{D} x \Delta_{\mu}^{2}(x) \Delta_{\nu}^{2}(x)= \\
& \quad=I_{D}-3 \omega^{2} \int d^{D} x \Delta^{2}(x) \Delta_{\mu}^{2}(x) .
\end{aligned}
$$

We now make the important observation that the subtle integral $I_{D}$ of Eq. (45) appears in Eqs. (46), (54) and (55) in such a way that it drops out from the sum of the watermelon diagrams in (20):

$$
\begin{gathered}
\Theta+4 \Leftrightarrow+\Theta \\
=\int d^{D} x \Delta^{2}(x) \Delta_{\mu \mu}^{2}(x)+\omega^{2} \int d^{D} x \Delta^{2}(x) \Delta_{\mu}^{2}(x) .
\end{gathered}
$$

Using (49) and (50), the right-hand side becomes a sum of completely regular expressions. Moreover, adding to this sum the last two watermelon-like diagrams in Eq. (20):

$$
4 \omega^{2} \bigoplus=4 \omega^{2} \int d^{D} x \Delta^{2}(x) \Delta_{\mu}^{2}(x),
$$

and

$$
\frac{2}{3} \omega^{4} \bigoplus=\frac{2}{3} \omega^{4} \int d^{D} x \Delta^{4}(x),
$$

we obtain for the contribution of all watermelon-like diagrams (20) the simple expression

$$
\begin{aligned}
-F_{2}^{(4)} & =-2 g^{2} \int d^{D} x \Delta^{2}(x) \\
\times\left[\Delta_{\mu \mu}^{2}(x)+5 \omega^{2} \Delta_{\mu}^{2}(x)+\frac{2}{3} \omega^{4} \Delta^{2}(x)\right] & \\
& =\frac{2}{3} \omega^{2} \Delta^{3}(0) \underset{D \rightarrow 1}{=} \frac{1}{12 \omega} .
\end{aligned}
$$

This cancels the finite contribution (33), thus making also the second-order free energy in (15) vanish, and confirming the invariance of the perturbatively defined path integral under coordinate transformations up to this order.

\section{SUMMARY}

In this note we have set up simple rules for calculating integrals over products of distributions in configuration space which produce the same results as dimensional regularization in momentum space. For a path integral of a quantum-mechanical point particle in a harmonic potential, we have shown that these rules lead to a reparametrization-invariant perturbation expansions of path integral.

Let us end with the remark that in the time-sliced definition of path integrals, reparametrization invariance has been established as long time ago in the textbook $[$.

[1] H. Kleinert and A. Chervyakov, Phys. Lett. B 464, 257 (1999) (hep-th/9906156).

[2] H. Kleinert and A. Chervyakov, Phys. Lett. B (2000) (in press) (quant-ph/9912056).

[3] G. 't Hooft and M. Veltman, Nucl. Phys. B 44, 189 (1972).

[4] H. Kleinert, Path Integrals in Quantum Mechanics, Statistics, and Polymer Physics, World Scientific, Singapore,

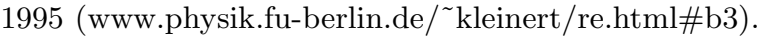

